\title{
Prevalence of lumbosacral transitional vertebrae in dogs in the Czech Republic
}

\author{
Iva Fialová ${ }^{1}$, Michaela Paninárová ${ }^{2}$, Alois Nečas ${ }^{1}$, Ladislav Stehlík $^{2}$, Pavel Proks $^{2}$ \\ ${ }^{1}$ University of Veterinary and Pharmaceutical Sciences Brno, Faculty of Veterinary Medicine, Small Animal \\ Clinic, Department of Surgery and Orthopaedics, ${ }^{2}$ Department of Diagnostic Imaging, Brno, Czech Republic \\ Received January 22, 2014 \\ Accepted July 23, 2014
}

\begin{abstract}
Lumbosacral transitional vertebra is a common congenital anomaly of the spine in dogs. It is a predisposing factor for degeneration of the lumbosacral spine and development of cauda equina syndrome or hip dysplasia in affected dogs. The aim of the study was to determine breed predisposition, types, and prevalence of lumbosacral transitional vertebrae in the canine population in the Czech Republic. The value of laterolateral radiographs of the lumbosacral junction in the diagnosis of LTV was also evaluated. Prevalence of lumbosacral transitional vertebrae was determined by reviewing ventrodorsal radiographs of pelvis with an extended hip of 1,878 dogs. Lumbosacral transitional vertebrae were detected in 188 dogs (10\%). German Shepherd, Alaskan Malamute and Bohemian Shepherd were found to be highly predisposed breeds. The most common type of lumbosacral transitional vertebra was type II with separation of the first sacral vertebra from sacrum and presence of rudimentary intervertebral space between S1 and the sacral median crest $(37.8 \%$ of the lumbosacral transitional vertebrae). Type I was detected in $29.2 \%$ and the asymmetric type of the lumbosacral transitional vertebra (type III) in 33\%. Laterolateral radiograph of the lumbosacral spine was evaluated in 126 dogs from 188 with lumbosacral transitional vertebrae. Rudimentary intervertebral disc space between S1 and S2 in laterolateral radiographs was detected in $100 \%$ of lumbosacral transitional vertebrae with type II and III, and was not detected in type I. The findings on lumbosacral transitional vertebrae in the Czech Republic will extend knowledge about the disease. Both ventrodorsal hip-extended and laterolateral radiographs should be recommended for routine screening and reliable differentiation among the three different types of lumbosacral transitional vertebra.
\end{abstract}

Hip dysplasia, cauda equina syndrome, radiography

Lumbosacral transitional vertebra (LTV) is a congenital abnormally formed vertebra between the last normal lumbar vertebra and the first normal sacral vertebra (Morgan 1968; Damur-Djuric et al. 2006). The LTV has the characteristics of both spinal segments, although definition varies in veterinary literature (Morgan 1968; Colter 1993; Morgan 1999; Damur-Djuric et al. 2006; Westworth and Sturges 2010). It is assumed that the LTV is a predisposing factor for earlier degeneration of lumbosacral junction and it is a risk factor for development of cauda equina syndrome (Lang et al. 1992; Morgan et al. 1993; Flückiger et al. 2006; Moore et al. 2001). The asymmetric type of LTV may result in pelvis rotation with a unilateral increase load of the hip and a higher degree of hip dysplasia (Flückiger et al. 2009). It is assumed that the occurrence of LTV has a hereditary condition, although the type of inheritance of LTV has not been elucidated yet (Flückiger et al. 2009). Prevalence of LTV in populations of dogs in different countries varies from 2.5 to $40.4 \%$ in dependence on the classification system (Morgan 1999; Damur-Djuric et al. 2006; Ledecký et al. 2007; Wigger 2009; Lappalainen et al. 2012).

To the authors' knowledge, no work has been published relating specifically to prevalence of LTV in pure breed dogs in the Czech Republic. The aim of this study was to determine the prevalence of lumbosacral transitional vertebrae in the dog population in

Address for correspondence:

MVDr. Iva Fialová

Small Animal Clinic

University of Veterinary and Pharmaceutical Sciences Brno

Palackého tř. 1/3, 61242 Brno, Czech Republic 
the Czech Republic, and the value of laterolateral radiographs of lumbosacral junction in determination of LTV type.

\section{Materials and Methods}

Radiographs of dogs that were screened for hip dysplasia or other problems with pelvis or the hip joint were evaluated at the University of Veterinary and Pharmaceutical Sciences Brno in the years 2007-2013. Radiographs of 1,878 dogs (892 males and 986 females), were evaluated retrospectively for presence of LTV. Their age ranged from 7 months to 11 years (median 1.6 years). For each dog, a ventrodorsal radiograph was taken of the pelvis with the pelvic limbs extended. Additionally, in some dogs laterolateral radiographs of lumbosacral spine were available.

Two of the authors evaluated the radiographs together and assessed the morphology of the lumbosacral spine. The occurrence and type of LTV and the breed were classified by consensus of the evaluators. One of them is certified reviewer. Prevalence of LTV was evaluated individually in 24 breeds with a minimum of 15 dogs per breed. Breeds with less than 15 dogs per breed were classified as "other breeds".

We used LTV evaluation according to Flückiger et al. (2009) as a basic classification scheme: type 0 is a normally shaped section of the lumbosacral spine. Type I of LTV in dogs is separated spinous process of the first sacral vertebra (S1) from the medial crest without signs of rudimental intervertebral space between S1and the rest of the sacrum. The LTV II is completely separated from sacrum; transverse processes are symmetrical and can be in a different attachment to the pelvis. Type III is an asymmetric type of LTV; transverse processes have different shapes (Flückiger et al. 2009).

The LL radiograph of the lumbosacral spine was evaluated for presence of intervertebral space between the first and second sacral vertebrae. Presence of intervertebral space was evaluated subjectively as the presence of radiolucent line representing the intervertebral disc. A $\chi^{2}$-test was used for statistical analysis of LTV in relation to sex.

\section{Results}

Out of a total of 1,878 evaluated dogs, LTV was identified in $188 \mathrm{dogs}(10 \%)$ of which $101(53.7 \%)$ were males and $87(46.3 \%)$ were females. In 62 cases $(33.0 \%)$ LTV was diagnosed solely using the VD projection. In 126 cases $(67.0 \%)$ LTV was diagnosed using both VD and LL projection. The group of dogs with LTV included 60 breeds. Prevalence of LTV varied from 1.9 to $25.9 \%$ among breeds with 15 or more dogs per breed (Table 1).

In the Czech Republic, breeds with the highest prevalence of LTV were German Shepherd with 53 patients (25.9\%), 7 Bohemian Shepherd dog (43.7\%) and 6 Alaskan Malamute $(22.2 \%)$.

Of 188 dogs with LTV, type I was detected in 55 dogs (29.2\%), including 28 dogs of the German Shepherd breed. Of the 55 dogs with a separate spinous process on the VD radiograph, less than half of the dogs $(40.0 \%)$ were accompanied by a LL view. Rudimentary intervertebral space in 58 of the type II dogs was confirmed in 58 LL projections $(100 \%)$. Type III included 62 patients (33\%), 10 of them were German Shepherds (18.7\%).

Rudimental intervertebral space between LTV I, or LTV II, and the rest of the sacrum was always visible from laterolateral projection and was not assessable from ventrodorsal projection.

\section{Discussion}

Prevalence of LTV in the dog population depends primarily on the LTV classification used and on presence of different breeds in the population. Different classification schemes vary in morphological evaluation criteria, in the number of classification levels, and in the number of radiographs necessary to classify the type of LTV (Damur-Djuric et al. 2006; Wigger et al. 2009; Flückiger et al. 2009; Lappalainen et al. 2012).

In order to classify LTV in dogs in our study, Flückiger's method which is also used for monitoring LTV in Switzerland (Flückiger et al. 2009) was used. We did not use the method of Lappalainen et al. (2012), who measured the length of the last lumbar vertebra 
Table 1. Prevalence of lumbosacral transitional vertebrae in dogs in the Czech Republic.

\begin{tabular}{|c|c|c|c|c|c|}
\hline \multirow{2}{*}{ Breed } & \multirow{2}{*}{ Number of dogs (n) } & \multirow{2}{*}{ Dogs with LTV (\%) } & \multicolumn{3}{|c|}{ Type of LTV (n) } \\
\hline & & & $\mathrm{I}$ & II & III \\
\hline Bohemian Shepherd & 16 & $7(43.7 \%)$ & 5 & 2 & 0 \\
\hline German Shepherd & 205 & $53(25.9 \%)$ & 28 & 15 & 10 \\
\hline Alaskan Malamute & 27 & $6(22.2 \%)$ & 2 & 3 & 1 \\
\hline American Pit Bull Terrier & 15 & $3(20.0 \%)$ & 1 & 0 & 2 \\
\hline Poodle & 21 & $4(19.0 \%)$ & 1 & 1 & 2 \\
\hline Rhodesian Ridgeback & 89 & $14(15.7 \%)$ & 1 & 9 & 4 \\
\hline White Swiss Shepherd & 37 & $5(13.5 \%)$ & 1 & 3 & 0 \\
\hline Caucasian Shepherd & 16 & $2(12.5 \%)$ & 0 & 1 & 0 \\
\hline Cocker Spaniel & 32 & $4(12.5 \%)$ & 1 & 1 & 2 \\
\hline Border Collie & 50 & $6(12.0 \%)$ & 3 & 2 & 1 \\
\hline Gordon Setter & 19 & $2(10.5 \%)$ & 0 & 2 & 0 \\
\hline Samoyed & 21 & $2(9.5 \%)$ & 1 & 1 & 0 \\
\hline Hungarian Pointer & 51 & $4(7.8 \%)$ & 1 & 0 & 3 \\
\hline Tosa-Inu & 27 & $2(7.4 \%)$ & 0 & 2 & 0 \\
\hline Rottweiler & 68 & $5(7.4 \%)$ & 0 & 2 & 3 \\
\hline Great Dane & 36 & $2(5.6 \%)$ & 0 & 1 & 1 \\
\hline Central Asian Shepherd Dog & 20 & $1(5.0 \%)$ & 0 & 1 & 0 \\
\hline Fila Brasileiro & 21 & $1(4.8 \%)$ & 0 & 1 & 0 \\
\hline American Staffordshire Terrier & 22 & $1(4.5 \%)$ & 0 & 0 & 1 \\
\hline Cane Corso & 24 & $1(4.2 \%)$ & 0 & 0 & 1 \\
\hline Golden Retriever & 133 & $5(3.8 \%)$ & 1 & 1 & 3 \\
\hline German Boxer & 37 & $1(2.7 \%)$ & 1 & 0 & 0 \\
\hline Bernese Mountain Dog & 38 & $1(2.6 \%)$ & 0 & 0 & 1 \\
\hline Hovawart & 51 & $1(1.9 \%)$ & 0 & 0 & 1 \\
\hline 36 other breeds & 802 & $55(6.9 \%)$ & 47 & 48 & 26 \\
\hline Total dogs of different breeds & 1878 & $188(10.0 \%)$ & 55 & 71 & 62 \\
\hline
\end{tabular}

LTV - lumbosacral transitional vertebrae

before LTV because we did not have always the whole length of this lumbar vertebra available on the x-ray.

In our study we found LTV prevalence in the population of purebred dogs in the Czech Republic to be $10.0 \%$. Prevalence of LTV in previously published studies ranged from 2.5 to $40.4 \%$. Comparing LTV prevalence in a population of dogs with various classification schemes is difficult. The LTV prevalence in the population of dogs in the Czech Republic is higher than in previous studies conducted in the USA, where the prevalence was $2.5 \%$ (Morgan 1999), and Switzerland (3.5\%) (Damur-Djuric et al. 2006). This is probably due to using different classification schemes, mostly by adopting the scheme with separation of the spinous process of S1 $(25.5 \%)$ as one of the types of LTV.

The occurrence of LTV in German Shepherds (25.9\%) in the Czech Republic is close to the results in other current German and Finnish studies. In Germany (Wigger 2009), the prevalence of LTV for German Shepherds is similar to the Czech Republic (28.2\%), probably due to also including the separated spinous process into type I unlike other studies (Larsen 1977; Damur-Djuric et al. 2006), where LTV prevalence for this breed was 
significantly lower. Conversely, higher prevalence was recorded in Finland (Lappalainen et al. 2012), probably due to the inclusion of dogs with eight lumbar vertebrae (11\%) in the group of LTV patients.

Differences in the results of prevalence in some breeds in other studies may be due to different national preferences (Flückiger et al. 2006; Damur-Djuric et al. 2006). The next commonly LTV-affected breeds in the Czech Republic were Bohemian Shepherd $(43.7 \%)$ and Alaskan Malamute (22.2\%). The high prevalence of Alaskan Malamutes affected with LTV in our study could be due to the fact that several of the evaluated dogs came from the same litter. The effect of heredity on LTV occurrence is highly suspected but so far no study has confirmed it (Flückiger et al. 2009).

Our study has also shown that LTV has no sex predisposition, which is in agreement with the results of other studies (Larsen 1977; Winkler 1985; Morgan 1999; Damur-Djuric et al. 2006; Ledecký et al. 2007).

In some cases a LL radiograph of the lumbosacral spine was added. Previously published classification schemes have low correlation between LTV classification from radiographs and CT scans because the sacrum can be seen without the superimposition of other pelvic structures using CT (Damur-Djuric et al. 2006; Lappalainen et al. 2012). Laterolateral projection can be appropriate for differentiation between types I and II (Ledecký et al. 2007).

Laterolateral radiograph seems to be a viable additional screening because particularly symmetric LTV are either easily overlooked or incorrectly classified. Misinterpretation occurs especially in cases where bone changes are minimal and symmetrical or when GIT or prepucium overlap the lumbosacral connection (Larsen 1977; Morgan et al. 1993; Breit et al. 2003). The transitional vertebra is best visible on ventrodorsal (VD) radiographs of the pelvis with pelvic limbs in extension (Breit et al. 2003) but LL radiographs may be more meaningful in some cases for proper evaluation of rudimental intervertebral space between LTV (S1) and the rest of the sacrum (Morgan 1968; Colter 1993; Morgan 1999; Damur-Djuric et al. 2006). Our study has shown that the evaluation of presence of rudimental intervertebral space between S1 and S2 can not be correctly evaluated without LL projections, unlike the identification of separated spinous process S1, where VD view is sufficient. Rudimental intervertebral space between S1 and S2 is easily overlooked in VD projection due to the diagonal placement of rudimental space towards radiograph surface. Therefore, the intervertebral space in LTV type III is visible on a VD radiograph, but it is narrower compared to the LL radiograph. In human medicine, this problem has been solved in Ferguson's radiograph (Konin and Walz 2010). In veterinary medicine a comparable solution is to perform LL radiographs of the lumbosacral spine.

Routinely, lumbosacral transitional vertebra is identified from a VD radiograph of the pelvis, most often performed as a part of checking for hip dysplasia by the OFA/FCI standard (Orthopaedic Foundation for Animals/Fédération Cynologique Internationale), and often as a secondary finding. When intermediate vertebrae are found, especially a separated spinous process S1, examination should be completed with laterolateral projections to evaluate the presence of separation of S1 from the rest of the sacrum. Our findings were in accordance with the study by Ledecký et al. (2007). In some countries, increasing attention is paid to transition vertebrae in dogs, preferring simple classification (Flückiger et al. 2009; Lapplainen et al. 2012). Clubs requiring evaluation of hip dysplasia are beginning to focus on the detection of LTV. Screening of genetic influence on LTV prevalence was started in Bern and Zurich (Flückiger et al. 2009). We assume that other countries including the Czech Republic will follow this trend.

Acknowledgement

This study was supported by IGA VFU Brno (project 75/2013/FVL). 


\section{References}

Breit S, Knaus I, Kunzel W 2003: Differentiation between lumbosacral transitional vertebrae, pseudolumbarisation, and lumbosacral osteophyte formation in ventrodorsal radiographs of the canine pelvis. Vet J 165: 36-42

Colter SB 1993: Congenital anomalies of the spine. In: Bojrab MJ (Ed.): Disease Mechanisms in Small Animal Surgery. Philadelphia, USA: Lippincott Williams and Wilkins, pp. 950-959

Damur-Djuric N, Steffen F, Hassig M, Morgan JP, Flückiger MA 2006: Lumbosacral transitional vertebrae in dogs: classification, prevalence, and association with sacroiliac morfology. Vet Radiol Ultrasound 47: 32-38

Flückiger MA, Damur-Djuric N, Hassig M, Morgan JP, Steffen F 2006: A lumbosacral transitional vertebra in the dog predisposes to cauda equine syndrome. Vet Radiol Ultrasound 47: 39-44

Flückiger M, Geissbuhler U, Lang J 2009: Lumbosacral transitional vertebra: what do they mean for the health of affected dogs? (in German). Schweiz Arch Tierheilkd 151: 133-135

Konin GP, Walz DM 2010: Lumbosacral transitional vertebrae: classification, imaging findings, and clinical revalence. Am J Neuroradiol 31: 1778-1786

Lang J, Hani H, Schawalder P 1992: A sacral lesion resembling osteochondrosis in the German Shepherd dog. Vet Radiol Ultrasound 33: 69-79

Lappalainen AK, Salomaa R, Junnila J, Snellman M, Laitinen-Vapaavuori O 2012: Alternative classification and screening protocol for transitional lumbosacral vertebra in German shepherd dogs. Acta Vet Scand 54: 27

Larsen JS 1977: Lumbosacral transitional vertebrae in the dog. Vet Radiol 18: 76-79

Ledecký V, Gjengedal H, Skurková L, Hluchý M, Ševčík A, Ledecká K 2007: Lumbosacral transitional vertebra in some dog breeds. Folia Vet 51:146-150

Moore GE, Burkman KD, Carter MN, Peterson MR 2001: Causes of death or reasons for euthanasia in military working dogs: 927 cases (1993-1996). J Am Vet Med Assoc 219: 209-214

Morgan JP 1968: Congenital anomalies of the vertebral column of the dog: a study of the incidence and significance based on a radiographic and morphologic study. J Am Vet Rad Soc 9: 21-29

Morgan JP, Bahr A, Franti CE, Bailey CS 1993: Lumbosacral transitional vertebrae as a predisposing cause of cauda equina syndrome in German Shepherd dogs: 161 cases (1987-1990). J Am Vet Med Assoc 202: 1877-1882

Morgan JP 1999: Transitional lumbosacral vertebral anomaly in the dog: a radiographic study. J Small Anim Pract 40: $167-172$

Westworth RD, Sturges KB 2010: Congenital spinal malformations in small animals. Vet Clin Small Anim 40: 951-981

Wigger A, Julier-Franz C, Tellhelm B, Kramer M 2009: Lumbosacral transitional vertebrae in German Shepherd dogs: incidence, forms, genetics, and correlation with hip dysplasia (in German). Tierärztl Prax 29: 7-13

Winkler W 1985: Lumbosacral transitional vertebrae in dogs (in German). Thesis in Veterinary Medicine, Freie Universität, Berlin. 